\title{
XXXI. Measuring-instruments used in mechanical testing
}

\author{
Prof. W.C. Unwin F.R.S.
}

To cite this article: Prof. W.C. Unwin F.R.S. (1887) XXXI. Measuring-instruments used in mechanical testing , Philosophical Magazine Series 5, 23:142, 282-286, DOI: 10.1080/14786448708628006

To link to this article: http://dx.doi.org/10.1080/14786448708628006

曲 Published online: 29 Apr 2009.

Submit your article to this journal $₫$

Џ Article views: 3

Q View related articles $₫$ 
which shows a tolerably close correspondence with the results obtained by the writer. A more complete discussion of Peirce's results is reserved until the relation between "No. 3 " and $S_{1}^{\alpha}$ and $S_{2}^{a}$ shall be more exactly known. The latter standards would appear to be the more trustworthy, since they are based on various independent determinations; while "No. 3" is based on an indirect comparison with metre "No. 49," a standard concerning the exact length of which there seems to be some little doubt.

\section{Measuring-Instruments used in Mechanical Testing.} $B y$ Prof. W. U. Unwin, F.R.S.*

TVHE determination of the exact distance between two fine marks on a standard of length is an operation of some difficulty, as is well known to physicists. But that operation is free from many of the difficulties which attend the measurements which have to be made in the engineering laboratory. Among these the determination of the modulus of elasticity (Young's modulus) of a bar by measuring its change of length by stress is one of the most important. Now the bars subjected to test are usually, in the part which can be measured, not more than 10 inches in length; and the whole elastic extension of such a bar is generally only about 0.007 inch. It is obvious, therefore, that measurements must be made with considerable accuracy and refinement to be of any value. But the bar cannot be placed in a position convenient for measurement; and the attachments to the testing-machine are more or less in the way of the measuring-apparatus to be applied. The bar itself is a somewhat rough bar, the form of which must not be interfered with to facilitate the measurements. Then also bars of very different forms have to be tested, flat and round, of variuus widths and diameters; and the measuring-apparatus must be applicable to all these with equal readiness. Last, but not least, the work of an engineering laboratory is pressing, and measurements must be carried out with rapidity.

In some cases, two diamond scratches have been made on the bar, and the distance between these measured by two micrometer-microscopes. Apparatus of this kind is awkward to apply on the testing-machine, and tedious to adjust and read.

A cathetometer has been used. But then two adjustments

* Communicated by the Physical Society : read January 22, 1887. 
have to be made, and two readings taken for each elongation. Also the limit of accuracy of the cathetometer is hardly sufficient for the purpose.

Very often mechanical magnification by a lever is adopted. But there are some difficulties in satisfactorily attaching a lever-apparatus to the bar : if a leverage of 100 to 1 is adopted, the fulcrum distance becomes very short, and the range of the apparatus is limited. There is also some difficulty in the calibration of the instrument to determine the value of the readings.

A micrometer-screw is sometimes used as a means of mechanical magnification. With this there is, again, the difficulty of suitable attachment to the bar; and, as generally used, it is difficult to ascertain when exact contact of the screw is obtained without excessive pressure.

There is a special difficulty in measuring the elongation of ordinary test-bars which has been overlooked in the construction of most of the apparatus of this kind. It is difficult to get test-bars which are rigidly straight. Even if the test-bar is strictly straight, it is difficult to hold it in the testingmachine, so that the resultant of the stress on any cross section passes strictly through the centre of figure of the section. Now if this condition is not satisfied, the bar becomes curved during the test. The straightening of an initially curved bar, or the curving of an initially straight one, introduce errors in the measurements of very considerable amount.

If the measurements could be made at the axis of the bar, the errors of this kind with any amount of curvature likely to occur would not be very serious; but this is of course impossible. The best that can be done is to measure at the surface of the test-bar. But, in straightening, the surface of the bar on one side lengthens and on the other shortens, and thus introduces a not inconsiderable error of measurement. If, as in many forms of elongation measuring-apparatus, the measuring-points are two inches or more from the axis of the bar, the errors become very large relatively to the elongations to be measured.

Let fig. 1 represent a bar bent in the plane of the paper, the centre of curvature being 0 . Then, if measurements could be made on the axis of the bar, between the points $a b$, the straightening of the bar would introduce an error equal to the difference of the length of the chord $a b$ and arc $a c b$. With any amount of curvature likely to occur in a test-bar, this error would not be very serious. Generally, however, the best that can be done is to measure the distance between points $a_{1} b_{1}$ on the surface of the bar. Then, since by 
straightening the lines $a \mathrm{O}, b \mathrm{O}$ become parallel, the error introduced is the difference between $a_{1} b_{1}$ and the are $a c b$;

Fig. 1.

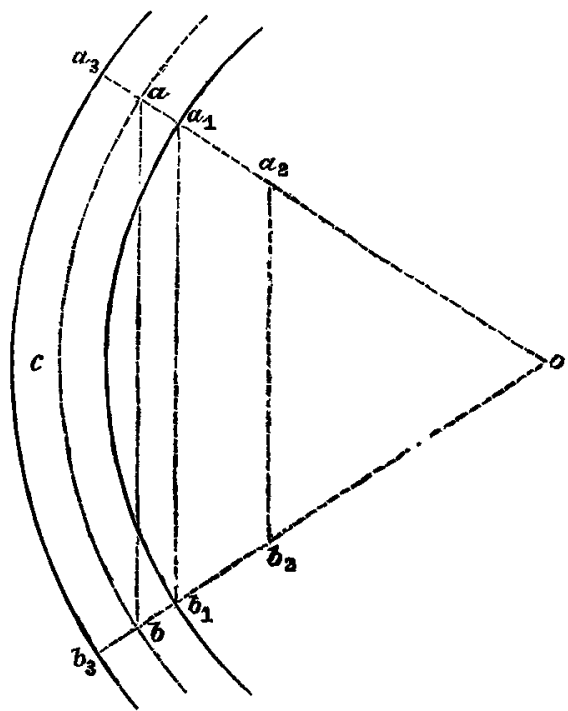

and this is much more serious. Most commonly, however, measurements are made between points on clips fastened to the bar at 1 or 2 inches distance from its surface, such as $a_{2} b_{2}$. Then the error introduced by straightening is the difference between $a_{2}, b_{2}$ and the arc $a c b$; and this may be a serious error, even with a very small amount of initial curvature.

If simultaneous measurements are taken of $a_{1} b_{1}$ and $a_{3} b_{3}$, the mean of these will have no greater error than the measurement of $a b$. That is, the mean of measurements on two sides of the bar reduces the error due to initial or induced curvature to the same amount as a measurement actually made at the axis of the bar.

Prof. Bauschinger, of Munich, appears to have been the first to recognize the importance of this double measure ment symmetrically on the two sides of the bar. He has always used an apparatus in which a finger, or touch-piece, attached to one end of the bar, presses on a roller attached to the other end. As the bar extends, the roller rotates by friction ag ainst the finger. A mirror is attached to the roller ; and the am ount 
of rotation is observed by noting the image of a scale in the mirror through a reading-telescope. In this way measurements to $\frac{1}{125000}$ th of an inch can be taken. To eliminate errors due to curvature, two rollers are placed, one on each side of the bar, and two sets of readings are taken. This involves the adjustment of two instruments and the taking of two sets of readings. But the principle is perfect; and no more accurate measurements than Bauschinger's have probably been made.

Touch-Micrometer Extensometer.-The first instrument used by the author was a kind of callipers. Two bars, one sliding in the other, could be set by tonch to the distance between two fixed clips on the test-bar. A scale was engraved on silver on one bar ; and the distance of the nearest division from a fixed zero-mark on the other was taken by a microscopemicrometer. Readings could be taken to $\frac{1}{10000}$ th of an inch. The instrument is easy and rapid to use. Readings can be taken on both sides of the test-bar; and the readings are direct on to a carefully graduated scale, so that no calibration of the instrument is necessary.

Screw-Micrometer Extensometer.-This aims at obtaining the extension along the axis of the bar by a single reading. Two clips are fixed on the bar, each by a pair of steel points, one on each side, gripping the bar in a plane through its axis. If, then, these clips can be made to preserve the same relative position to the bar, the middle points of the clips will move in the same way as points on the axis of the bar. Fig. 2 is a diagrammatic sketch of the apparatus. $a a$ and $b b$ are the clips on the test-bar, fixed to it by points in its middle plane. $c c$ are projections on the clips, to which are fixed delicate spirit-levels; $d$ is a small screw which just touches the test-bar; $e$ is a micrometer-screw with graduated head, which supports the upper clip on the lower clip. In use the lower clip is first levelled by the screw $d$; then the upper clip is levelled by the micrometer-screw, and a reading taken. The clips being always accurately levelled, in a plane perpendicular to that in which the four points attaching the clips to the test-bar lie, the micrometer-readings are the distances between the middle points of the two parallel clips; and their differences are the mean of the elongations on the two sides of the test-bar, or virtually are readings at the axis of the test-bar. Readings to $\frac{1}{10000}$ th of an inch can be taken.

Roller-and-Mirror Extensometer.-.Fig 3 is a diagrammatic sketch of another instrument on the same principle. $a$ and $b$ are two cljps similar to those in fig. 2 ; the lower clip is sup- 
ported on the test-bar by a screw $d$; the upper clip is supported on the lower by a stay-bar with knife-edges, $e$. At $r$

Fig. 2 .

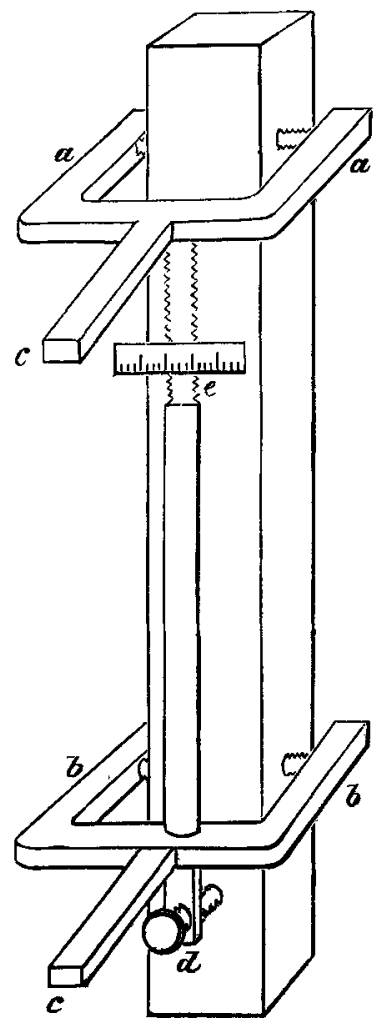

Fig. 3.

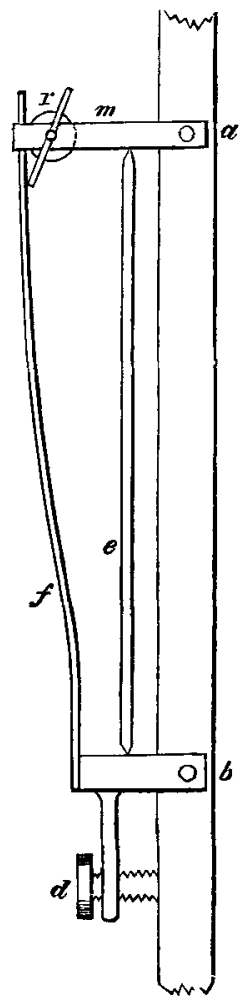

and $m$ are the roller and mirror, the axis of these being at the same distance from the knife-edge of the stay-bar as the set screw of the clip. A touch-piece or finger, $f$, attached to the lower clip presses on the roller. If the bar extends, the roller approaches the lower clip by an equal amount; it turns against the finger $f$; and the amount of rotation is read by a telescope and scale. This instrument will easily read to. $\frac{1}{100000}$ th of an inch. The roller being at the centre of the clip, its movement is the mean of the elongations on the two sides of the test-bar.

The author showed a third instrument on the same principle, for obtaining the compression of small blocks of stone. 